\title{
Hérnia perineal em cães
}

\author{
Perineal hernia in dogs
}

Ana Carolina Mortari ${ }^{1}$ Sheila Canevese Rahal ${ }^{2}$

\section{- REVISÃO BIBLIOGRÁFICA -}

\section{RESUMO}

A hérnia perineal acomete mais freqüentemente cães machos de meia idade e idosos, sexualmente intactos, podendo ser uni ou bilateral. Por tratar-se de afecção com altos índices de recidiva e complicações pós-operatórias, o trabalho tem por objetivo abordar os sinais clínicos, métodos diagnósticos, com ênfase aos tratamentos cirúrgicos. Entre as técnicas mais efetivas de reconstrução do diafragma pélvico, estão as transposições musculares únicas ou combinadas, tais como as do músculo obturador interno ou músculo glúteo superficial. Em casos de recidivas, podem ser utilizados métodos complementares como a colopexia e cistopexia por fixação dos ductos deferentes, ou procedimentos mais complexos como a transposição do músculo semitendinoso.

Palavras-chave: hérnia, períneo, canino, tratamento, cirurgia.

\section{ABSTRACT}

The perineal hernia occurs more frequently in middle aged to aged male dogs, sexually intact, and it may be unilateral or bilateral. Because this disease may have high rate of recurrence and postoperative complications, the aim of this paper is to describe the clinical signs, diagnosis, foccusing to surgical treatments. Among the most effective techniques of pelvic diaphragm reconstruction are the single or associated muscular transpositions, such as internal obturator muscle transposition or superficial gluteal muscle transposition. In cases of recurrences, complementary methods such as colopexy and cystopexy by deferent duct fixation, or more complex procedures as semitendinosus muscle transposition may be used.
Key words: hernia, perineum, canine, treatment, surgery.

\section{INTRODUÇÃO}

A hérnia perineal resulta do enfraquecimento e separação dos músculos e fáscias que formam o diafragma pélvico, promovendo deslocamento caudal de órgãos abdominais ou pélvicos no períneo (ANDERSON et al., 1998; SEIM III, 2004). A doença é comum em cães machos, especialmente os intactos, e rara em fêmeas (DIETERICH, 1975; ANDERSON et al., 1998). O intervalo de maior incidência está entre os sete e nove anos de idade, com poucos relatos antes de cinco anos (ANDERSON et al., 1998; BELLENGER \& CANFIELD, 2003). O processo pode ser uni ou bilateral, mas geralmente em casos unilaterais, o lado contralateral apresenta-se alterado (DIETERICH, 1975; RAISER, 1994).

Em geral, a hérnia perineal ocorre entre os músculos esfíncter externo do ânus e elevador do ânus e, ocasionalmente, entre os músculos elevador do ânus e coccígeo (BELLENGER \& CANFIELD, 2003). A causa exata da fraqueza muscular é desconhecida, mas alguns fatores têm sido propostos, como atrofia muscular neurogênica ou senil, miopatias, aumento de volume da próstata,

${ }^{1}$ Curso de Pós-graduação da Faculdade de Medicina Veterinária e Zootecnia, Unesp Botucatu, SP, Brasil. Email: ana_mortari@yahoo.com.br

${ }^{2}$ Departamento de Cirurgia e Anestesiologia Veterinária, Faculdade de Medicina Veterinária e Zootecnia, Unesp Botucatu. Rubião Júnior s/n, 18618000, Botucatu, SP, Brasil. Email: sheilacr@fmvz.unesp.br. Autor para correspondência. 
alterações hormonais e constipação crônica (HEDLUND, 2002; BELLENGER \& CANFIELD, 2003; SEIM III, 2004). Algumas raças apresentam predisposição, tais como o boston terrier, o pequinês e o boxer (HAYES et al., 1978; SEIM III, 2004). Por tratar-se de afecção com altos índices de recidiva e complicações pós-operatórias, o trabalho tem por objetivo abordar os sinais clínicos, métodos diagnósticos, com ênfase aos tratamentos cirúrgicos.

Sinais clínicos e diagnóstico

Os sinais clínicos mais citados são tenesmo, constipação e aumento de volume perineal, que pode ser redutível ou não (HOSGOOD et al., 1995; ANDERSON et al., 1998; SEIM III, 2004). Se houver retroflexão da bexiga urinária, ocorrerão estrangúria, disúria e anúria (ANDERSON et al., 1998; BELLENGER \& CANFIELD, 2003). Vários conteúdos são encontrados no saco herniário, sendo comum a presença de fluido seroso (DIETERICH, 1975). Além disso, podem ser observados: bexiga urinária; próstata; saculação, dilatação, flexura, desvio ou divertículo retal; e nódulos de coloração creme a vermelho-marrom (HOSGOOD et al., 1995; BELLENGER \& CANFIELD, 2003). Estes últimos são hematomas consolidados ou fragmentos de gordura retroperitoneal em processo de necrose avascular e reabsorção (DIETERICH, 1975; BELLENGER \& CANFIELD, 2003).

O diagnóstico baseia-se na história clínica, sinais clínicos, bem como exames físicos, radiográficos e ultra-sonográficos (ANDERSON et al., 1998; HEDLUND, 2002; BELLENGER \& CANFIELD, 2003; SEIM III, 2004). A palpação retal é um dos exames mais importantes, visto possibilitar a determinação das estruturas que formam o aumento de volume, verificar a presença de deslocamento ou dilatação retal, e avaliar a textura e tamanho da próstata, se esta estiver envolvida (DIETERICH, 1975; BELLENGER \& CANFIELD, 2003). Radiografias não contrastadas podem indicar a posição da bexiga urinária, próstata, bem como deslocamento e dilatações retais, desde que o reto esteja preenchido por fezes (HEDLUND, 2004). Quando a bexiga urinária não pode ser visibilizada em exames radiográficos rotineiros, realiza-se a uretrografia retrógrada ou a cistografia (ANDERSON et al., 1998). A ultra-sonografia é efetiva na determinação dos conteúdos herniários, dispensando muitas vezes o exame radiográfico (BELLENGER \& CANFIELD, 2003). Estudos eletroneuromiográficos podem ser úteis para determinar lesões nervosas e avaliar o acometimento da musculatura do diafragma pélvico antes do procedimento cirúrgico (SJOLLEMA et al., 1993).

Tratamentos cirúrgicos

Existe uma grande variedade de procedimentos cirúrgicos, porém serão abordados os considerados mais exeqüíveis pelos autores da presente revisão. Entre os tratamentos mais freqüentemente utilizados na reconstrução do diafragma pélvico estão: o método tradicional de sutura; a transposição do músculo obturador interno, com ou sem secção do tendão muscular; a transposição do músculo glúteo superficial; e a transposição do músculo obturador interno aliado à transposição do músculo glúteo superficial (RAFFAN, 1993; ANDERSON et al., 1998; HEDLUND, 2002; BELLENGER \& CANFIELD, 2003). Se houver anormalidades retais associadas (desvio, saculação ou divertículo), estas devem ser corrigidas conjuntamente com a herniorrafia (KRAHWINKEL, 1983; MANN, 1993). Quando a hérnia é bilateral, as lesões podem ser corrigidas conjuntamente ou com intervalo de quatro a seis semanas entre os procedimentos cirúrgicos (ANDERSON et al., 1998). Em casos de recidivas, podem ser utilizadas técnicas de colopexia e a cistopexia por fixação dos ductos deferentes ou a técnica de transposição do músculo semitendinoso (BILBREY et al., 1990; MANN \& CONSTANTINESCU, 1998).

A orquiectomia é recomendada em associação às diversas técnicas cirúrgicas de tratamento da hérnia perineal, em especial por seus efeitos benéficos nas doenças prostáticas, testiculares ou neoplasias da glândula perineal (BELLENGER, 1980; WEAVER \& OMAMEGBE, 1981; HARDIE et al., 1983; SJOLLEMA \& VAN SLUIJS, 1989; RAISER, 1994; HOSGOOD et al., 1995), sendo esta sempre utilizada pelos autores da presente revisão. Apesar desta conduta ser controversa, há referências entre sua não utilização e alta taxa de recorrência (HAYES et al., 1978; ANDERSON et al., 1998). Vale referir que a castração não previne o enfraquecimento da musculatura do diafragma pélvico (ANDERSON et al., 1998).

Antes do procedimento cirúrgico de reconstrução do diafragma pélvico, o reto deve ser esvaziado e a bexiga urinária cateterizada com sonda uretral (ANDERSON et al., 1998). Se for necessário o uso de enema, esse deve ser realizado aproximadamente 18 horas antes da intervenção, para permitir a evacuação total de líquidos e evitar a contaminação local (MUÑOZ et al., 2000). Antibióticos profiláticos efetivos contra bactérias gram-negativas e anaeróbias são administrados por via venosa em seguida à indução anestésica (HEDLUND, 2002). 
Após tricotomia de toda região perineal e porção caudal dos membros pélvicos, o cão deve ser mantido em decúbito esternal, com os membros pélvicos colocados fora da mesa cirúrgica, porém protegidos com material macio para evitar injúria dos nervos isquiáticos (MATTHIESEN, 1989; MANN \& CONSTANTINESCU, 1998) e femorais (DIETERICH, 1975). A cauda é posicionada em direção à cabeça e fixada por meio de esparadrapo de forma a ficar na linha média da região torácica dorsal. A mesa cirúrgica deve ser inclinada para frente, mantendo o animal em posição perineal (VAN SLUIJS \& SJOLLEMA, 1989; ANDERSON et al., 1998; HEDLUND, 2002). Antes da anti-sepsia, o ânus deve ser ocluído com uma sutura em bolsa de tabaco (RAISER, 1994; ANDERSON et al., 1998).

O reconhecimento anatômico das estruturas que compõem o períneo é essencial para a realização dos diferentes procedimentos cirúrgicos. $\mathrm{O}$ períneo é circundado dorsalmente pela terceira vértebra caudal, pelos ligamentos sacrotuberosos em ambos os lados e pelo arco do ísquio ventralmente (ANDERSON et al., 1998). A fossa isquiorretal é delimitada pelos músculos esfíncter externo do ânus, coccígeo e elevador do ânus medialmente, pelo músculo obturador interno ventralmente e pela parte caudal do músculo glúteo superficial lateralmente (MUÑOZ et al., 2000). O diafragma pélvico (Figura 1) consiste no fechamento vertical do canal pélvico através do qual passa o reto, sendo composto pelos músculos coccígeo e elevador do ânus, e fáscias interna e externa (ANDERSON et al., 1998; BELLENGER \& CANFIELD, 2003). A artéria pudenda interna, a veia pudenda interna e o nervo pudendo correm juntos pela porção ventrolateral do músculo coccígeo e continuam caudalmente sobre a superfície dorsal do músculo obturador interno (MANN \& CONSTANTINESCU, 1998; HEDLUND, 2002).

\section{Método tradicional de suturas}

Realiza-se a incisão de pele sobre o aumento de volume perineal iniciando lateral à base da cauda até o ângulo medial da tuberosidade isquiática (BELLENGER \& CANFIELD, 2003). Após abertura do saco herniário, os conteúdos pélvicos e abdominais são identificados e recolocados na posição original ou removidos, se necessário (ANDERSON et al., 1998; HEDLUND, 2002; SEIM III, 2004). O método tradicional inclui basicamente suturas entre os músculos esfíncter externo do ânus e coccígeo, e entre os músculos esfíncter externo do ânus e obturador interno (ROBERTSON, 1984; MUÑOZ et al., 2000). Também podem ser incluídos os músculos coccígeo e elevador do ânus, mas este último muitas vezes se encontra atrofiado e não pode ser utilizado para ancorar pontos de sutura (ROBERTSON, 1984) (Figura 2). O ligamento sacrotuberal, que é geralmente palpado e não visualizado, estende-se desde a região do sacro até a tuberosidade isquiática, sendo utilizado para reforçar os pontos de sutura, juntamente com o músculo esfíncter externo do ânus com ou sem o músculo coccígeo (ROBERTSON, 1984; ANDERSON et al., 1998). Após o fechamento do defeito, a fáscia perineal superficial pode ser usada para reforçar a sutura (ANDERSON et al., 1998). O tecido subcutâneo e pele são aproximados e a sutura em bolsa de tabaco removida (WEAVER \& OMAMEGBE, 1981). O reto deve ser palpado para observar se suturas não foram colocadas inadvertidamente através da parede retal (ANDERSON et al., 1998). Apesar da simplicidade, é um dos métodos com altos índices de complicações, variando de 28,6 a 61\% (BURROWS \& HARVEY, 1973), e taxas de recorrência que oscilam entre 10\% (PETIT, 1962) e 46\% (BURROWS \& HARVEY, 1973), sendo por isso utilizado pelos autores da presente revisão apenas nos casos em que a musculatura esteja bem evidenciada.

Transposição do músculo obturador interno

A técnica de transposição do músculo obturador interno tem por objetivo o reforço na porção ventral da hérnia (ORSHER, 1986; BELLENGER \& CANFIELD, 2003). O procedimento também permite uma sutura sem tensão e promove mínima distorção do músculo esfíncter externo do ânus (HARDIE et al., 1983; ORSHER, 1986). Após acessar a hérnia de forma similar à técnica anterior, a porção caudal do músculo é elevada subperiostealmente do assoalho isquiático e o seu tendão pode (ROBERTSON, 1984; VAN SLUIJS \& SJOLLEMA, 1989) ou não ser seccionado (HEDLUND, 2002; SEIM III, 2004).

Quando o tendão não é seccionado, o músculo é apenas deslocado dorsalmente para fechar o defeito e estabilizado por suturas com o músculo esfíncter externo do ânus medialmente e ligamento sacrotuberal e músculo coccígeo lateralmente (MANN \& CONSTANTINESCU, 1998), como ilustrado na figura 3. Este procedimento é o mais utilizado pelos autores da presente revisão tanto no tratamento inicial como nos casos de recidiva pelo método tradicional de suturas.

Se realizada a secção do tendão, segundo VAN SLUIJS \& SJOLLEMA (1989), deve-se tomar cuidado para não lesar o nervo pudendo que se localiza dorsalmente a ele. O músculo coccígeo é suturado ao músculo esfíncter externo do ânus em pontos isolados 


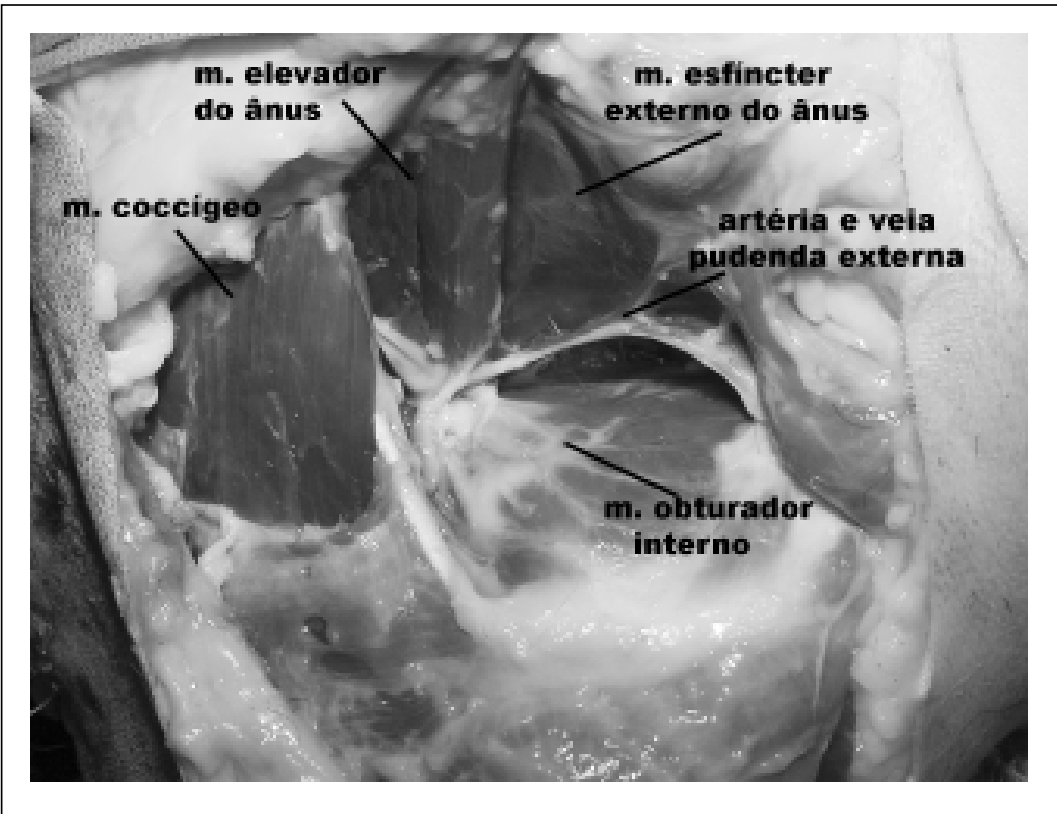

Figura 1 - Anatomia cirúrgica dos músculos que compõem o diafragma pélvico do cão.

o diafragma pélvico. Apesar de não induzir uma ferida cirúrgica muito extensa, o procedimento requer maior tempo cirúrgico, o que possibilita maior susceptibilidade à infecção (SPREUL \& FRANKLAND, 1980). As complicações estão entre 15\% (SPREUL \& FRANKLAND, 1980) e $58 \%$ e as recidivas em torno de 36\% (WEAVER \& OMAMEGBE, 1981).

Transposição do músculo semitendinoso

A transposição do músculo semitendinoso (Figura 4) pode ser particularmente utilizada quando a face ventral do períneo está severamente afetada (hérnias crônicas, recidivantes), assim como em hérnias perineais bilaterais (CHAMBERS \& RAWLINGS,

simples. Em seguida, aplica-se um ponto U separado incluindo os músculos obturador interno, coccígeo e esfíncter externo do ânus. O procedimento é finalizado aproximando-se os músculos obturador interno e esfíncter externo do ânus com pontos simples isolados.

As complicações pós-operatórias oscilam entre 19\% (HARDIE et al., 1983) e 45\% (VAN SLUIJS \& SJOLLEMA, 1989) e as taxas de recorrência entre 2,38\% (HARDIE et al., 1983) e 18,75\% (ORSHER, 1986).

Transposição do músculo glúteo superficial

A transposição do músculo glúteo superficial pode ser utilizada nos casos em que o tratamento tradicional falhou (SPREUL \& FRANKLAND, 1980) ou para reforço dorsal da hérnia (SPREUL \& FRANKLAND, 1980; MUÑOZ et al., 2000). Diferente do citado anteriormente, o cão deve ser posicionado em decúbito lateral (SPREUL \& FRANKLAND, 1980). O procedimento consiste em dissecar a borda cranial do músculo e seccionar sua inserção no terceiro trocanter do fêmur. O retalho muscular é refletido em ângulo de $90^{\circ}$ e suturado ao músculo esfíncter externo do ânus caudalmente e ao tecido subjacente dorsal e ventralmente (BELLENGER \& CANFIELD, 2003). Para a obtenção de um diafragma mais resistente, RAFFAN (1993) recomendou a associação desta técnica com a de transposição do músculo obturador interno.

A transposição do músculo glúteo superficial promove suporte seguro e duradouro para
1991; MANN \& CONSTINESCU, 1998). Quando a hérnia for unilateral utiliza-se o músculo semitendinoso contralateral na reconstrução (MANN \& CONSTANTINESCU, 1998).

A incisão de pele utilizada na herniorrafia tradicional é prolongada através da linha mediana em direção à tuberosidade isquiática, onde é curvada e progride distalmente no aspecto caudal do membro pélvico contralateral até a altura do linfonodo poplíteo (CHAMBERS \& RAWLINGS, 1991) (Figura 4a). O músculo semitendinoso é dissecado das estruturas adjacentes e seu pedículo proximal, vascular (artéria glútea caudal e veia glútea caudal) e nervoso, é identificado e preservado (CHAMBERS \& RAWLINGS, 1991; MORTARI, 2004) (Figura 4b). Após a ligadura da artéria femoral caudal distal, o músculo semitendinoso é seccionado na altura do linfonodo poplíteo (MORTARI, 2004). A extremidade seccionada do músculo semitendinoso é suturada aos músculos coccígeo e obturador interno e fáscia pélvica (CHAMBERS \& RAWLINGS, 1991; MORTARI, 2004). A borda medial original do músculo semitendinoso é aproximada à porção caudal do músculo esfíncter externo do ânus e a sua borda lateral original aos músculos isquiouretral, e bulboesponjoso, assim como fáscia do músculo semimembranoso (MANN \& CONSTANTINESCU, 1998; MORTARI, 2004). Suturas de apoio podem ser colocadas nos músculos do diafragma pélvico do lado contralateral para evitar tensão na porção do músculo semitendinoso que foi 


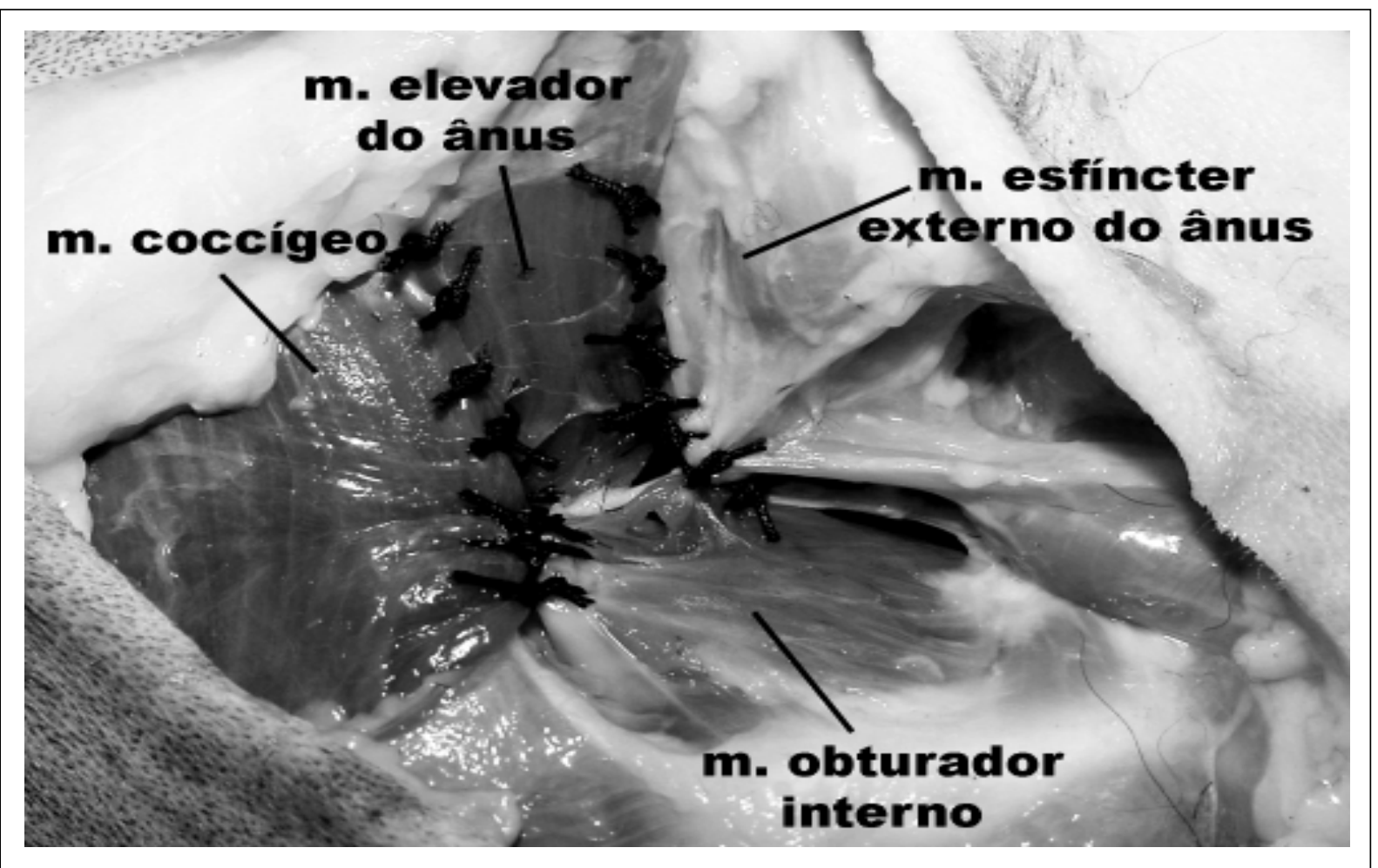

Figura 2 - Demonstração da reconstrução do diafragma pélvico de cão pelo método tradicional de suturas. Pontos interrompidos simples aplicados entre os músculos: esfíncter externo do ânus e obturador interno, esfíncter externo do ânus e elevador do ânus, coccígeo e elevador do ânus, e coccígeo e obturador interno.

utilizada para correção da hérnia (CHAMBERS \& RAWLINGS, 1991; MORTARI, 2004) (Figura 4c).

Cães submetidos experimentalmente ao procedimento não apresentaram prejuízos na locomoção ou movimentação articular no membro operado (CHAMBERS, 1999; MORTARI, 2004). Por outro lado, foi verificado que o músculo transposto manteve-se contrátil por 90 dias de acordo com os exames eletromiográficos, porém sofreu processo atrófico detectado em análises ultra-sonográficas e morfológicas (MORTARI, 2004).

Uma das maiores complicações observadas com esta técnica é o acúmulo de secreção associada ou não à deiscência de pontos (MORTARI, 2004), que pode ser reduzido com a utilização de drenos de suç̧ão ou de Penrose (CHAMBERS \& RAWLINGS, 1991; CHAMBERS, 1999).

Colopexia e cistopexia por fixação dos ductos deferentes

As técnicas de colopexia e cistopexia por fixação dos ductos deferentes são indicadas para prevenir a herniação dos órgãos mais acometidos (cólon, bexiga urinária e próstata) e podem ser adotadas quando não se obteve sucesso na reconstrução por transposições musculares (MANN \& CONSTANTINESCU, 1998; HEDLUND, 2002; BELLENGER \& CANFIELD, 2003). Os autores da presente revisão as empregam como complemento a transposição do músculo obturador interno ou do músculo semitendinoso, em especial nos casos de recidiva.

No procedimento de colopexia, após a exposição dos órgãos abdominais via celiotomia na linha média caudal, o cólon deve ser localizado, tracionado cranialmente e sua parede suturada à parede abdominal paramediana esquerda utilizando seis a oito pontos simples separados (MANN \& CONSTANTINESCU, 1998). Para induzir aderência mais intensa, realiza-se uma incisão na camada seromuscular da borda antimesentérica do cólon e outra correspondente da serosa ao músculo transverso do abdome. Em seguida, as margens das incisões são justaspostas e suturadas (BELLENGER \& CANFIELD, 2003).

Em casos de cães não castrados, para a realização da deferopexia, faz-se inicialmente a orquiectomia por via pré-escrotal, antes da celiotomia na linha média (MANN \& CONSTANTINESCU, 1998; BILBREY et al., 1990). Os ductos deferentes devem ser gentilmente tracionados através do canal inguinal 


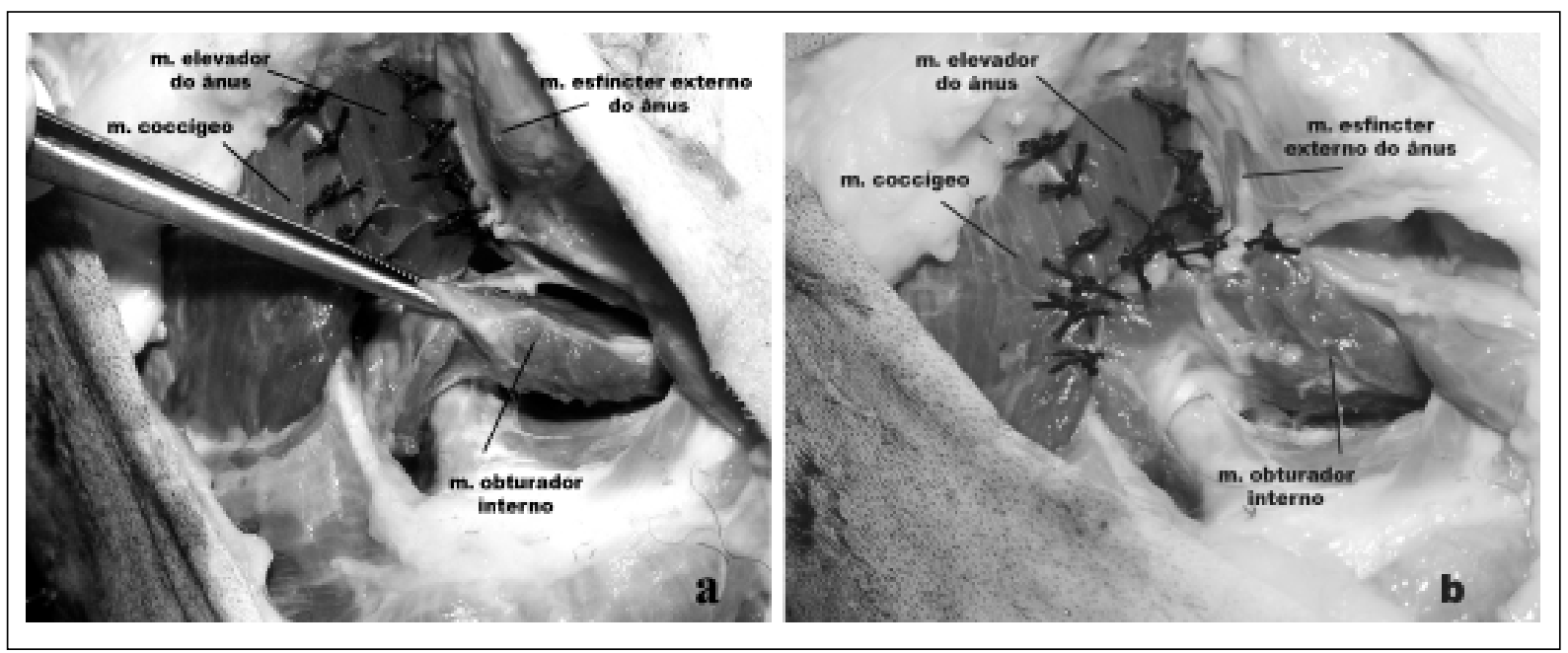

Figura 3 - Demonstração da reconstrução do diafragma pélvico de cão pela transposição do músculo obturador interno sem secção do tendão. (a) Músculo obturador interno elevado do assoalho pélvico e pontos isolados simples aplicados entre os músculos esfíncter externo do ânus e elevador do ânus, e entre os músculos coccígeo e elevador do ânus. (b) Músculo obturador interno suturado aos músculos coccígeo, elevador do ânus e esfíncter externo do ânus.

até que fiquem inteiramente livres (HEDLUND, 2002). Um túnel para a passagem do ducto, com cerca de 1 a $2 \mathrm{~cm}$ de comprimento, é criado na parede abdominal lateral por meio de duas incisões paralelas no músculo transverso abdominal e dissecadas com auxílio de uma pinça hemostática (BILBREY et al., 1990; MANN \& CONSTANTINESCU, 1998). Após ligeira tração, o ducto é passado através do túnel e sua porção livre é voltada caudalmente e suturada sobre si mesmo com pontos simples separados. A sutura pode ser reforçada utilizando pontos na parede abdominal (BILBREY et al., 1990; MANN \& CONSTANTINESCU, 1998). O procedimento deve ser realizado bilateralmente (HEDLUND, 2002).

\section{Cuidados pós-operatórios}

A utilização de antibióticos após 12 horas do procedimento cirúrgico é apenas indicada em pacientes debilitados ou com presença de tecidos isquêmicos, contaminados ou necróticos (HEDLUND, 2002; SEIM III, 2004). A ferida cirúrgica deve ser mantida limpa, e analgésicos e antiinflamatórios podem ser empregados para minimizar a dor e reduzir o edema (RAFFAN, 1993; HEDLUND, 2002; MORTARI, 2004). Situações de tenesmo devem ser controladas com uso de dietas ou laxantes para evitar esforço abdominal (MUÑOZ et al., 2000; HEDLUND, 2002). O cão deve ser mantido com colar protetor até a retirada de pontos (MUÑOZ et al., 2000; SEIM III, 2004).

Complicações

Muitas são as complicações observadas após o reparo de hérnias perineais. Entre elas podem ser citadas a lesão do nervo isquiático ou a do pudendo, a incontinência fecal, a infecção no local da incisão, a deiscência de suturas, a colocação de suturas no lúmen retal ou sacos anais, a necrose da vesícula urinária, a incontinência urinária, bem como a recorrência da hérnia (MATTHIESEN, 1989; ANDERSON et al., 1998; MUÑOZ et al., 2000; HEDLUND, 2002).

A lesão do nervo isquiático apresenta ocorrência menor que 5\% (MATTHIESEN, 1989), e está associada a acidentes no momento da colocação de sutura ao redor do ligamento sacrotuberal (MATTHIESEN, 1989; MANN \& CONSTANTINESCU, 1998; SEIM III, 2004). Além disso, a colocação do cão na mesa cirúrgica em posição perineal, com os membros pélvicos firmemente amarrados para frente, pode gerar neuropraxia ciática posicional devido à excessiva tensão ou isquemia (MATTHIESEN, 1989).

Incontinência fecal, temporária ou permanente, ocorre em cerca de $10 \%$ dos casos (BELLENGER \& CANFIELD, 2003), podendo ser causada por dano aos nervos pudendo ou caudal retal, ou por lesão direta ao músculo esfíncter externo do ânus durante a dissecação cirúrgica (BURROWS \& HARVEY, 1973; MATTHIESEN, 1989). Prolapsos retais aparecem com freqüência de 2 a $13 \%$ (LIPOWITZ, 1996), sendo fatores predisponentes a colocação de suturas no lúmen retal, dor, doenças retais prévias e lesão nervosa por manipulação cirúrgica (MATTHIESEN, 1989; ANDERSON et al., 1998). 


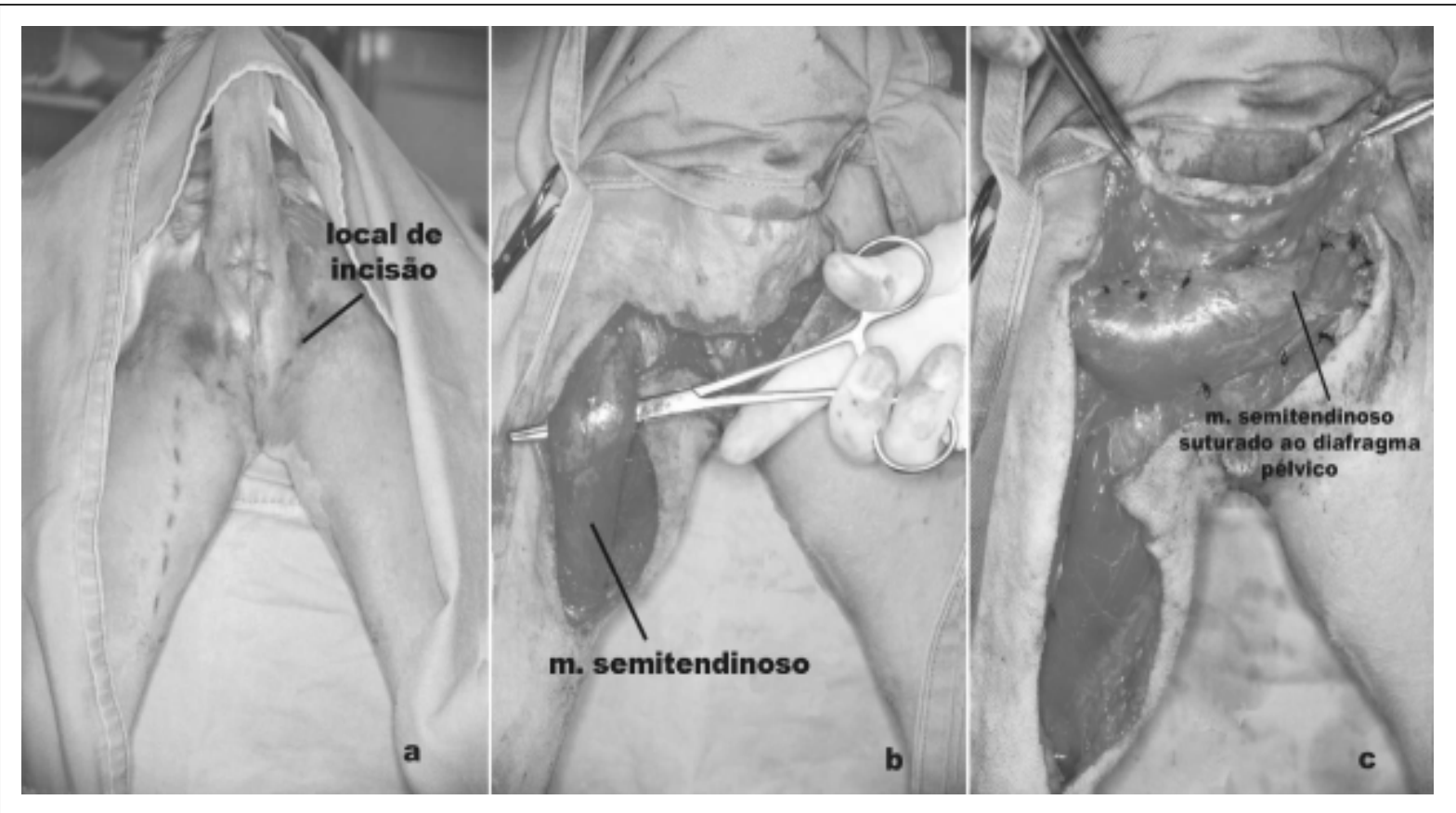

Figura 4 - Demonstração da técnica de transposição do músculo semitendinoso. (a) Demarcação da incisão de pele iniciando lateral à base da cauda até a rafe ventral, progredindo em direção à tuberosidade isquiática oposta, onde é curvada distalmente no aspecto caudal do membro pélvico até a altura do linfonodo poplíteo. (b) Dissecação do músculo semitendinoso. (c) Músculo transposto ao diafragma pélvico.

Necrose da bexiga urinária e incontinência urinária são complicações pós-operatórias incomuns e relacionadas à retroflexão da bexiga urinária, com prolongada distensão e obstrução (ANDERSON et al., 1998). Quadros de anúria são raros e geralmente resultantes de dano nervoso à bexiga urinária ou uretra proximal (LIPOWITZ, 1996). Hemorragias e lesões uretrais ocorrem por falha na identificação das estruturas anatômicas ou descuido no momento da dissecação dos tecidos (MATTHIESEN, 1989).

A formação de seroma deve ser diferenciada de infecção, abscesso ou recorrência de hérnia (LIPOWITZ, 1996), podendo ser prevenida pelo adequado posicionamento da fáscia perineal e tecido subcutâneo após a reconstrução do diafragma pélvico (LIPOWITZ, 1996). Infecção da ferida, deiscência ou formação de abscesso são problemas prevalentes, com taxa de ocorrência que variam de 6,4 a 26\% (MATTHIESEN, 1989; LIPOWITZ, 1996; BELLENGER \& CANFIELD, 2003). Entre os fatores predisponentes para a infecção, estão a contaminação fecal na ferida cirúrgica ou penetração do lúmen retal ou saco anal no momento da sutura, extensa dissecção ou estrangulação tecidual, hemorragia e falhas na anti-sepsia (MATTHIESEN, 1989).
As recidivas das hérnias perineais estão associadas à falha no isolamento das estruturas anatômicas, inadequada colocação de suturas ou escolha inapropriada de materiais de sutura (ROBERTSON, 1984). O tenesmo causado pela presença de divertículos e dilatações retais consiste em outro fator contribuinte para o processo (RAISER, 1994). A associação entre orquiectomia e recorrência tem sido referida como 2,5 vezes maior em cães não castrados; contudo, acredita-se que as falhas estão mais relacionadas à falta de familiaridade com o procedimento cirúrgico do que com o efeito da castração (ANDERSON et al., 1998). As taxas de recorrência variam conforme a técnica de correção utilizada, experiência do cirurgião, tempo de evolução da doença, conteúdo herniário e enfermidades associadas (ANDERSON et al., 1998; HEDLUND, 2002).

\section{CONCLUSÃO}

Os sinais clínicos mais freqüentes em cães com hérnia perineal são o tenesmo, a constipação e o aumento de volume perineal. Para um diagnóstico adequado, o exame físico deve incluir a palpação retal, podendo também ser necessária a realização de exames radiográficos e 
ultra-sonográficos. Entre as técnicas cirúrgicas de reconstrução do diafragma pélvico, as mais efetivas são as que utilizam transposições musculares únicas ou combinadas, tais como as do músculo obturador interno ou músculo glúteo superficial. Em casos de recidivas, empregam-se métodos complementares como a colopexia e a cistopexia por fixação dos ductos deferentes, ou procedimentos mais complexos, incluindo a transposição do músculo semitendinoso.

\section{REFERÊNCIAS}

ANDERSON, M.A. et al. Perineal hernia repair in the dog. In: BOJRAB, M.J. et al. Current techniques in small animal surgery. 4.ed. Baltimore : Williams \& Wilkins, 1998. Cap.35, p.555-564.

BELlENGER, C.R. Perineal hernia in dogs. Australian Veterinary Journal, Brunswick, v.56, n.9, p.434-438, 1980.

BELLENGER, C.R.; CANFIELD, R.B. Perineal hernia. In: SLATTER, D. Textbook of small animal surgery. 3.ed. Philadelphia : Saunders, 2003. Cap.34, p.487-498.

BILBREY, S.A. et al. Fixation of the deferent ducts for retrodisplacement of the urinary bladder and prostate in canine perineal hernia. Veterinary Surgery, Hagerstown, v.19, p.24-27, 1990 .

BURROWS, C.F.; HARVEY, C.E. Perineal hernia in the dog. Journal of Small Animal Practice, London, v.14, p.315331, 1973.

CHAMBERS, J.N. Pedicle muscles flaps. In:___. Manual of canine and feline wound management and reconstrution. Surrey : England, 1999. p.95-103, 152-153.

CHAMBERS, J.N.; RAWLINGS, C.A. Applications of a semitendinosus muscle flap in two dogs. Journal of the American Veterinary Medical Association, Shaumburg, v.199, n.1, p.84-86, 1991.

DIETERICH, H.F. Perineal hernia repair in the canine. Veterinary Clinics of North America Small Animal Practice, Philadelphia, v.5, n.3, p.383-399, 1975.

HARDIE, E.M. et al. Evaluation of internal obturator muscle transposition in treatment of perineal hernia in dogs. Veterinary Surgery, Hagerstown, v.12, n.2, p.69-72, 1983.

HAYES, H.M. et al. The epidemiologic features of perineal hernia in 771 dogs. Journal of the American Animal Hospital Association, v.14, p.703-707, 1978.

HEDLUND, C.S. Perineal hernia. In: FOSSUM, T.W. Small animal surgery. 2.ed. St. Louis : Mosby, 2002. p.433-437.

HEDLUND, C.S. Hérnia perineal: diagnóstico e tratamento. Focus, ed. esp., p.5-11, 2004.

HOSGOOD, G. et al. Perineal herniorrhaphy: perioperative data from 100 dogs. Journal of the American Animal Hospital Association, Goldon, v.31, n.4, p.331-341, 1995.
KRAHWINKEL Jr, D.J. Rectal diseases and their role in perineal hernia. Veterinary Surgery, Hagerstown, v.12, n.3, p.16091615, 1983.

LIPOWITZ, A.L. Perineal surgery. In: LIPOWITZ, A.L. et al. Complications in small animal surgery. Baltimore : Williams \& Wilkins, 1996. Cap.16, p.527-540.

MANN, F.A. Perineal herniation. In: BOJRAB, M.J. et al. Disease mechanisms in small animal surgery. 2.ed. Philadelphia : Lea \& Febiger, 1993. Cap.14, p.92-97.

MANN, F.A.; CONSTANTINESCU, G.M. Salvage techniques for failed perineal herniorrhaphy. In: BOJRAB, M.J. et al. (Eds). Current techniques in small animal surgery. 4.ed. Baltimore : Williams \& Wilkins, 1998. p.564-570.

MATTHIESEN, D.T. Diagnosis and management of complications occurring after perineal herniorraphy in dogs. Compendium on Continuing Education for the Practice Veterinary, Princeton Junction, v.11, n.7, p.797-803, 1989.

MORTARI, A.C. Avaliação da técnica de transposição do músculo semitendinoso no reparo do diafragma pélvico. Estudo experimental em cães. 2004. 101f. Dissertação (Mestrado) - Curso de Pós-graduação em Medicina Veterinária, Faculdade de Medicina Veterinária e Zootecnia, Universidade Estadual Paulista, Botucatu.

MUÑOZ, M.O. et al. Hernia perineal. Corboda : Universidad de Cordoba, 2000. Capturado em 09 de julho de 2004. On line. Disponível na Internet: http://www.uco.es/ organiza/departamentos/anatomia-y-anat.patologica/ pesques/.

ORSHER, R.J. Analysis of results of internal obturator transposition. Veterinary Surgery, Hargerstown, v.15, n.3, p.253-258, 1986.

PETTIT, G.D. Perineal hernia in the dog. Cornell Veterinarian, Ithaca, v.52, p.261-279, 1962.

RAISER, A.G. Heriorrafia perineal em cães - análise de 35 casos. Brazilian Journal of Veterinary Research and Animal Science, São Paulo, v.31, n.3/4, p.252-260, 1994.

RAFFAN, P.J. A new surgical technique for repair of perineal hernia in the dog. Journal of Small Animal Practice, London, v.34, n.13-19, 1993.

ROBERTSON, J.J. Perineal hernia repair in dogs. Modern Veterinary Practice, Santa Barbara, v.65, n.5, p.365-368, 1984.

SEIM III, H.B. Perineal hernia repair. In: WORLD CONGRESS IN SMALL ANIMAL VETERINARY MEDICINE, 29., 2004, Rhodes. Proceedings... Rhodes : Alta Grafico Publisher, 2004. V.1, p.833-836.

SJOLLEMA, B.E.; VAN SLUIJS, F.J. Perineal hernia repair in the dog by transposition of the internal obturator muscle. II Complications and results in 100 pacients. Veterinary Quartely, Dondrechet, v.11, n.1, p.18-23, 1989. 
SJOLLEMA, B.E. et al. Electromyography of the pelvic diaphragm and anal sphincter in dogs with perineal hernia. American Journal of Veterinary Research, Schaumburg, v.54, n.1, p.185-190, 1993.

SPREUL, J.S.A.; FRANKLAND, A.L. Transplanting the superficial gluteal muscle in the treatment of perineal hernia and flexure of the rectum in the dog. Journal of Small Animal Practice, London, v.21, p.265-278, 1980.
VAN SLUIJS, F.J.; SJOLLEMA, B.E. Perineal hernia repair in the dog by transposition of the internal obturator muscle. Surgery technique. Veterinary Quartely, Dondrechet, v.11, n.1, p.1317, 1989.

WEAVER, A.D.; OMAMEGBE, J.O. Surgical treatment of perineal hernia in the dog. Journal of Small Animal Practice, London, v.22, n.749-758, 1981 . 